\title{
Sinais radiológicos no tórax
}

\author{
Radiologic signs in the thorax \\ Danilo Tadao Wada ${ }^{1}$ (D), José Antonio Hiesinger Rodrigues ${ }^{1}$, Marcel Koenigkam Santos ${ }^{2}$
}

\begin{abstract}
RESUMO
Reconhecer os principais sinais radiológicos nos exames de tórax pode auxiliar na identificação dos padrões de doenças e estreitar o diagnóstico diferencial. Muitos sinais utilizam termos metafóricos e alguns são descritos até como altamente sugestivos de um diagnóstico. Alguns sinais podem ser vistos tanto na radiografia quanto na tomografia, enquanto outros apenas em um ou outro método. Juntamente com a semiologia radiológica correta e o uso adequado da terminologia, o conhecimento dos sinais radiológicos favorece a interpretação mais correta das alterações nos exames de imagem do tórax. Nesse artigo, realizamos uma breve revisão ilustrativa dos principais sinais descritos na radiografia do tórax.
\end{abstract}

Palavras-chave: Radiografia Simples. Tórax. Sinais Radiológicos.

\begin{abstract}
Knowledge of the main radiology signs may help recognizing patterns of diseases and cover the main possible differential diagnosis. Many of the signs use metaphoric terms and some are told highly suggestive of a specific disease. Some signs may be recognized in conventional chest radiography and in computed tomography, while others are just applicable in one of these methods. Along with the use of the correct semiology and terminology, knowledge of these signs helps to achieve the correct interpretation of chest radiography findings. In this article, we've made a brief review of the main thoracic radiology signs.
\end{abstract}

Keywords: Conventional Radiography. Chest. Signs.

1. Médico assistente da disciplina de Radiologia Torácica e Cardiovascular. Centro de Ciências das Imagens e Física Médica, Hospital das Clínicas da Faculdade de Medicina de Ribeirão Preto da Universidade de São Paulo, Ribeirão Preto (SP), Brasil.

2. Docente responsável pela disciplina de Radiologia Torácica e Cardiovascular. Fundação de Apoio ao Ensino, Pesquisa e Assistência do Hospital das Clínicas da Faculdade de Medicina de Ribeirão Preto da Universidade de São Paulo (FAEPA), Ribeirão Preto (SP), Brasil.

[M. Danilo Tadao Wada. Avenida Bandeirantes, 3900 - Campus Universitário. CEP: 14049-900. Ribeirão Preto SP, Brasil. dwada@hcrp.usp.br | Recebido em: 17/02/2019 | Aprovado em: 26/04/2019 


\section{INTRODUÇÃO}

W a prática clínica de avaliação dos exames de imagem do tórax utilizamos dos sinais radiológicos para auxiliar na identificação dos padrões de doenças e assim estreitar o diagnóstico diferencial $^{1,2}$. Desta maneira, quando identificamos um sinal radiológico, em geral, estamos acrescentando especificidade à nossa hipótese diagnóstica. Muitos sinais utilizam termos metafóricos e alguns são descritos até como altamente sugestivos de um diagnóstico. Boa parte dos sinais podem ser identificados tanto na radiografia simples (RX) quanto na tomografia computadorizada (TC) do tórax.

Juntamente com a semiologia radiológica correta e o uso adequado da terminologia, o conhecimento dos sinais radiológicos favorece a interpretação mais correta das alterações nos exames de imagem do tórax ${ }^{3}$.

Neste texto serão descritos os principais sinais radiológicos relacionados aos exames de $\mathrm{RX}$ do tórax. Os sinais estão ordenados por ordem alfabética.

\section{OBJETIVOS GERAIS}

Após a leitura deste texto, o leitor deverá ser capaz de:

- $\quad$ reconhecer os principais sinais radiológicos no tórax

- conhecer as principais doenças relacionadas a estes sinais

\section{Descrição dos sinais}

\section{$1-2-3$}

Alargamento mediastinal e hilar bilateral ${ }^{4-6}$ típico da sarcoidose, visto na radiografia em posteroanterior (PA). O sinal do 1-2-3 está relacionado à linfonodopatia da sarcoidose nas regiões paratraqueal direita e nos hilos pulmonares direito e esquerdo, nestes, caracteristicamente, de maneira simétrica (figura 1 ).

\section{Asa de borboleta}

Distribuição das opacidades alveolares ao redor dos hilos pulmonares na região central dos pulmões, de maneira simétrica, característico do edema alveolar pulmonar cardiogênico (edema agudo de pulmão) ${ }^{7-8}$ (figura 2 ).

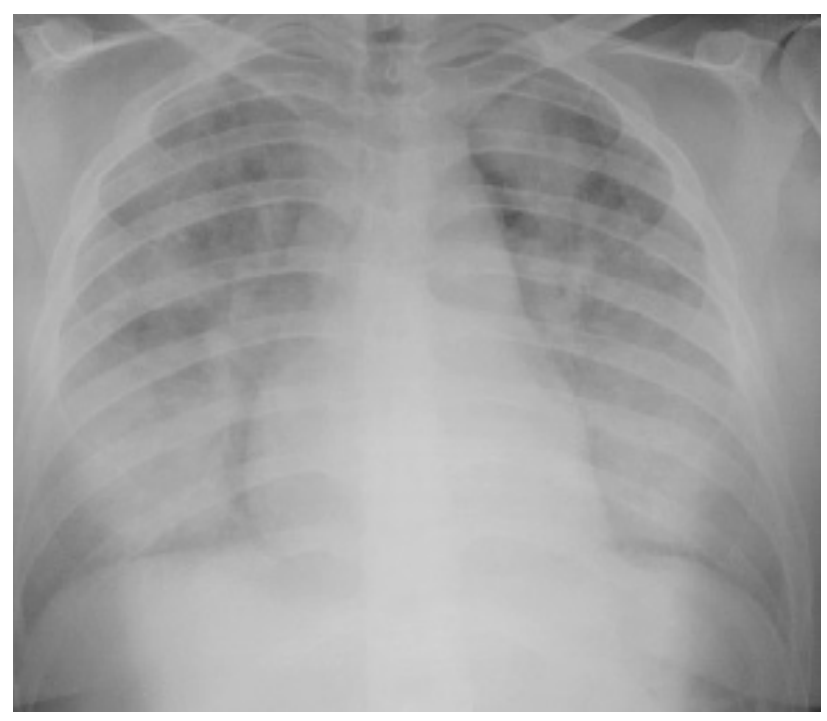

Figura 2: Sinal da asa de borboleta em paciente com edema agudo de pulmão.

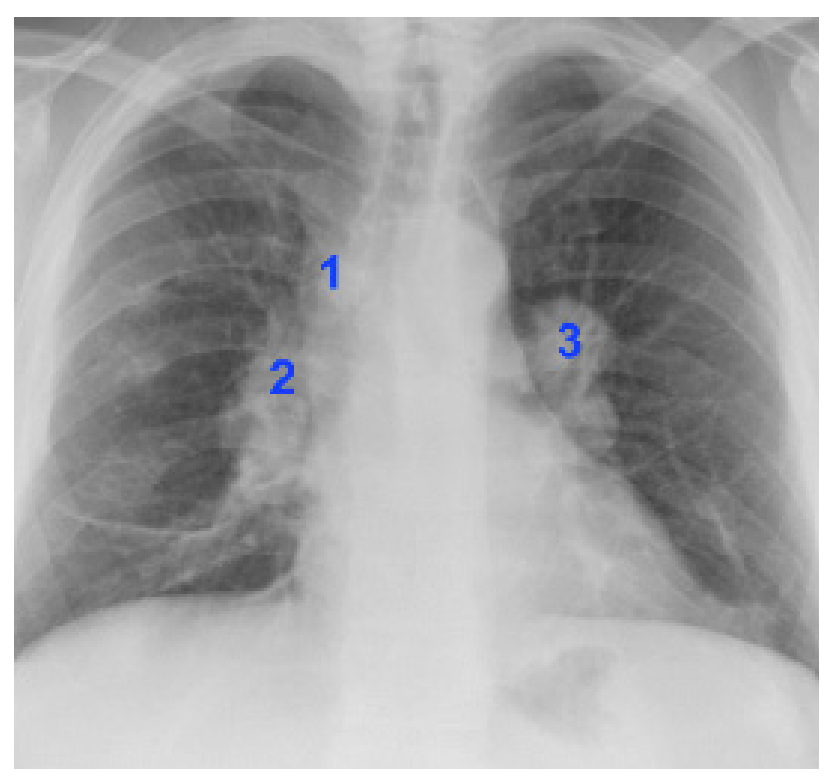

Figura 1: Sinal do 1-2-3 em paciente com sarcoidose e linfonodopatia mediastinal. 


\section{Borda incompleta}

Sinal que indica no RX que a opacidade mais provavelmente é extrapulmonar. Este sinal é identificado quando a margem da opacidade voltada para o pulmão é bem delimitada, enquanto a margem voltada para a parede torácica ou mediastino tem limites imprecisos ${ }^{9-11}$ (figura 3 ).

\section{Broncograma aéreo}

Um dos primeiros sinais radiográficos, representa os brônquios fisiológicos pérvios, com conteúdo aéreo, em meio a uma consolidação, as vezes relacionado a um nódulo, massa ou outra opacidade. O sinal do broncograma indica não somente que a alteração é pulmonar, mas também que está relacionada ao preenchimento alveo$\mid a r^{2,4,11}$. Mais comumente é visto na pneumonia e no edema, mas pode estar presente na atelectasia e mesmo em alguns tumores (adenocarcinoma pulmonar, doença linfoproliferativa) (figura 4).

\section{Cervicotorácico}

Sinal relacionado ao alargamento mediastinal na radiografia em $\mathrm{PA}$, indicando a presença de uma massa provavelmente do mediastino anterior. Se o alargamento mediastinal é mal definido acima do nível da clavícula, a lesão provavelmente é do mediastino anterior por ter contato (sinal da silhueta) com as partes moles cervicais s, $^{1,12,13}$. Quando a lesão é do mediastino posterior, em geral, tem limite bem definido acima do nível da clavícula (figura 3 ).

\section{Cimitarra}

A cimitarra é uma espada árabe grande e curvada. O sinal da cimitarra aparece como uma opacidade pulmonar alongada, curva e verticalizada, lembrando esta espada, nos pacientes com retorno venoso parcial anômalo a direita ${ }^{1,2,14}$ (figura 5).

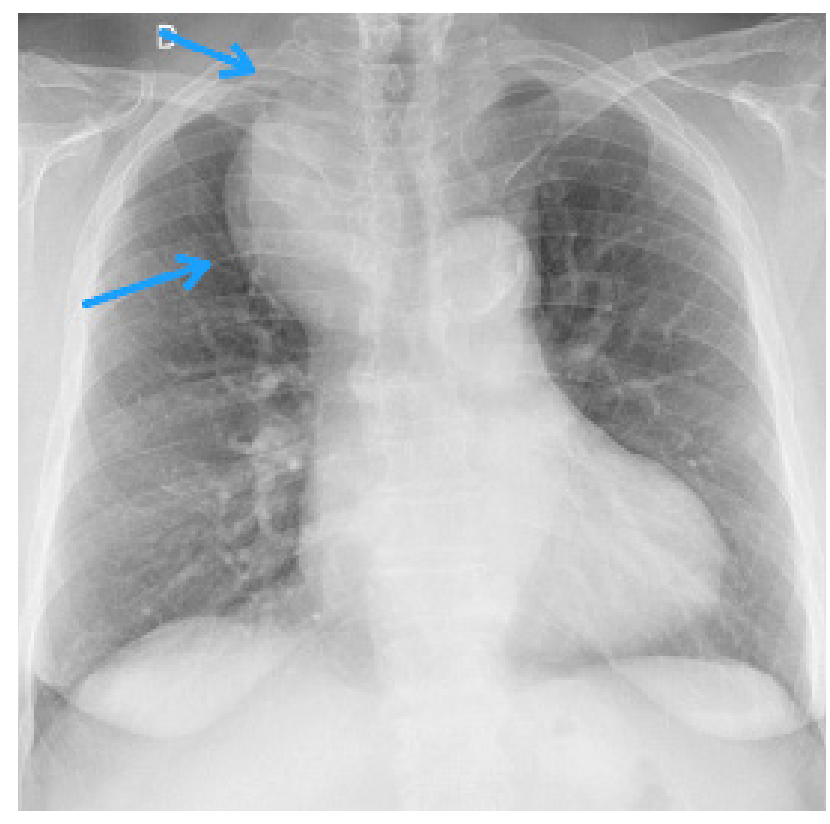

Figura 3: Sinal da borda incompleta em paciente com bócio mergulhante. Neste caso, observa-se também o sinal cervicotorácico.

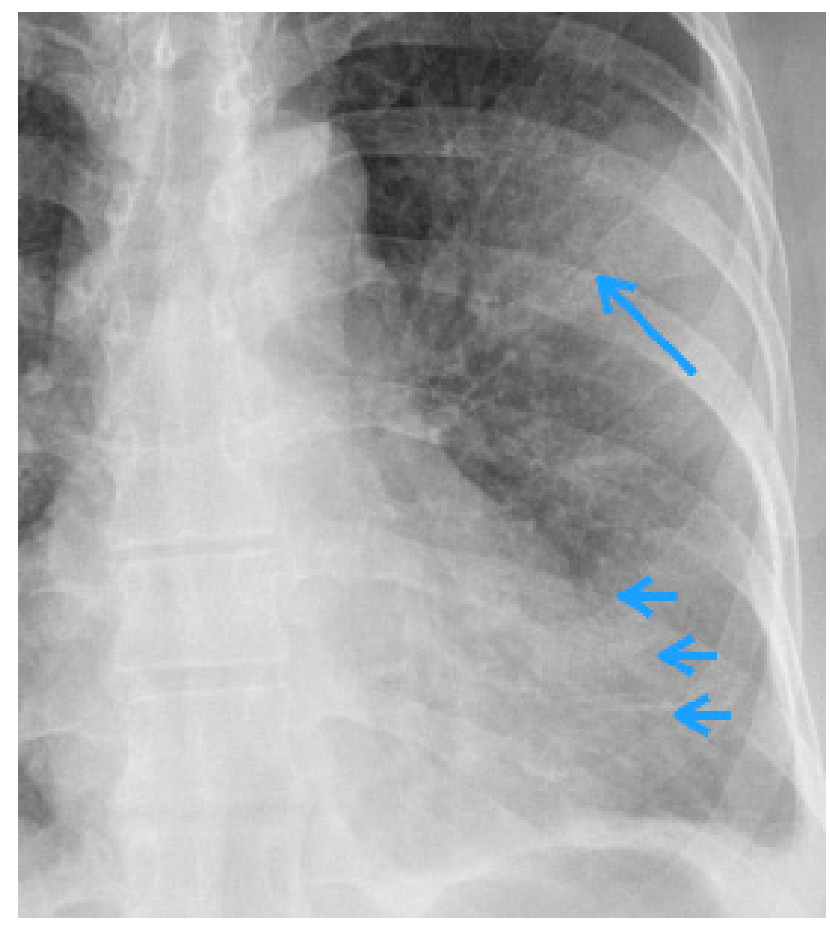

Figura 4: Paciente com opacidades alveolares incluindo consolidações no pulmão esquerdo, apresentando sinal do broncograma aéreo (seta longa) e sinal da silhueta (setas curtas) com o contorno cardíaco esquerdo. 


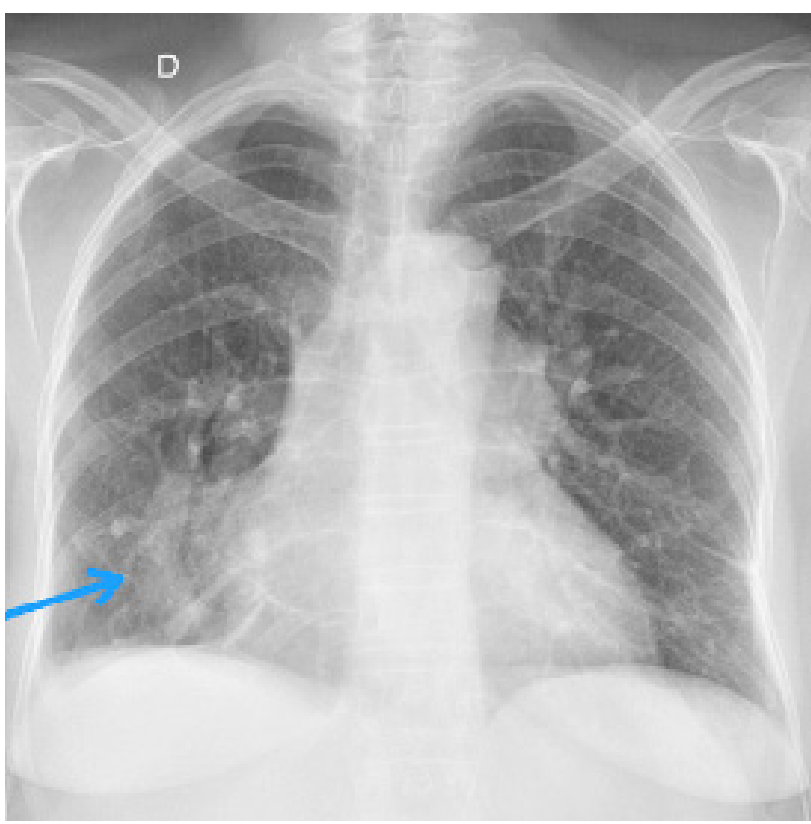

Figura 5: Sinal da cimitarra em paciente com retorno venoso parcial anômalo a direita.

\section{Convergência hilar}

Neste sinal identifica-se que o hilo pulmonar está aumentado de tamanho e com morfologia alterada, ou seja, a alteração está relacionada a estrutura ou tecido componente do hilo. Diferentemente do sinal da sobreposição hilar (Iesão extra-hilar), na convergência hilar não se individualizam as estruturas normais do hilo pulmonar $^{1,4,11}$ (figura 6).

\section{Crescente aéreo}

Relacionado a um nódulo ou massa, sendo característico da presença da bola fúngica (fungo Aspergillus) no interior de uma cavidade, bronquiectasia ou outra lesão de conteúdo aéreo. Este sinal é identificado mais comumente na região apical e indica a presença do aspergiloma no interior de uma cavidade sequelar de tuberculose. A linha externa ao crescente aéreo representa a parede da cavidade, enquanto a linha interna delimita a bola fúngica propriamente dita ${ }^{1,15,16}$ (figura 7).

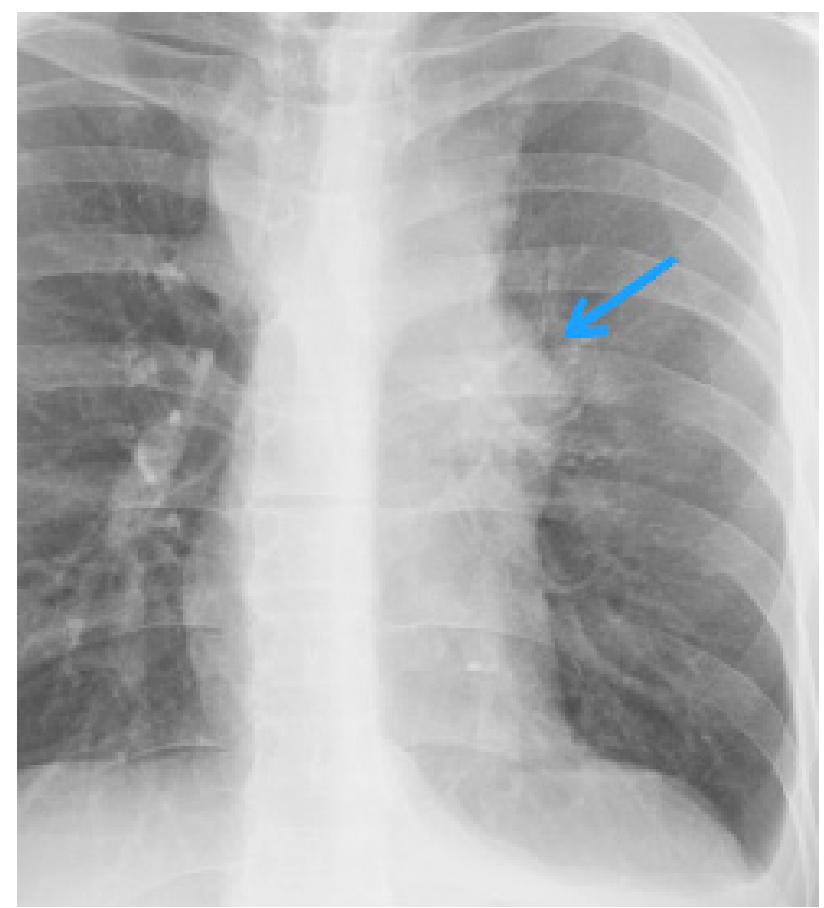

Figura 6: Paciente com linfoma, alargamento mediastinal e opacidade peri hilar esquerda, com sinal do hilo convergente por linfonodomegalia hilar.

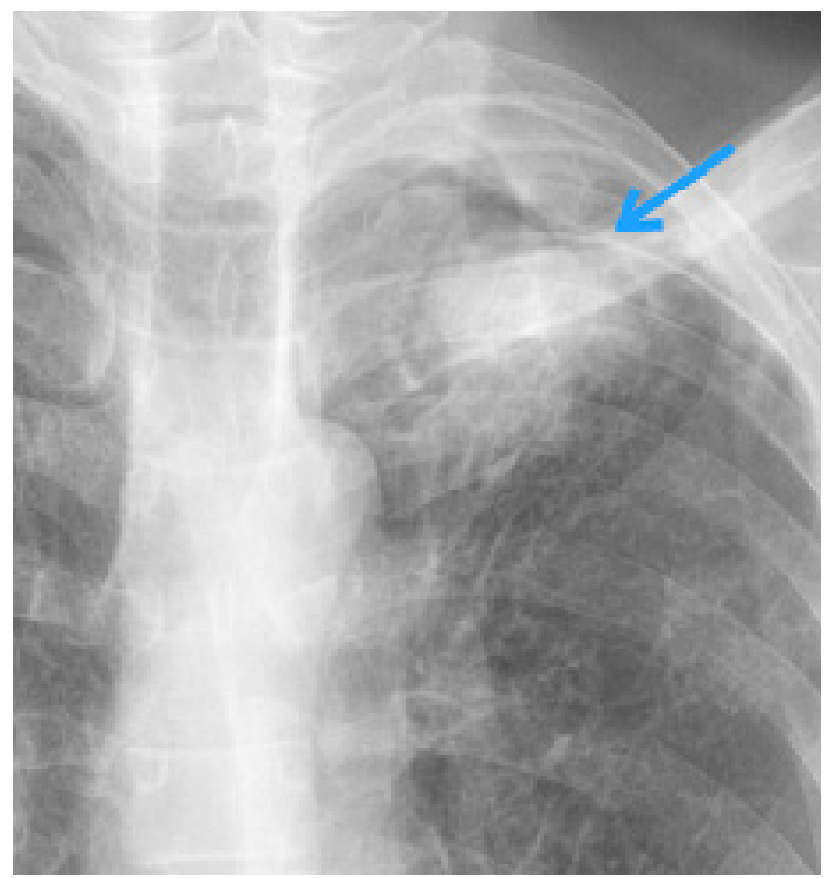

Figura 7: Sinal do crescente aéreo em paciente com bola fúngica no interior de cavidade de tuberculose prévia no ápice do pulmão esquerdo. 


\section{Corcova de Hampton}

Sinal radiográfico que representa 0 infarto pulmonar secundário ao tromboembolismo pulmonar agudo. É caracterizado por uma opacidade periférica de base pleural em forma de cunha, na base pulmonar adjacente ao diafragma ${ }^{1,2,17,18}$ (figura 8). O nome é homenagem a Aubrey Otis Hampton (1900-1955), radiologista americano.

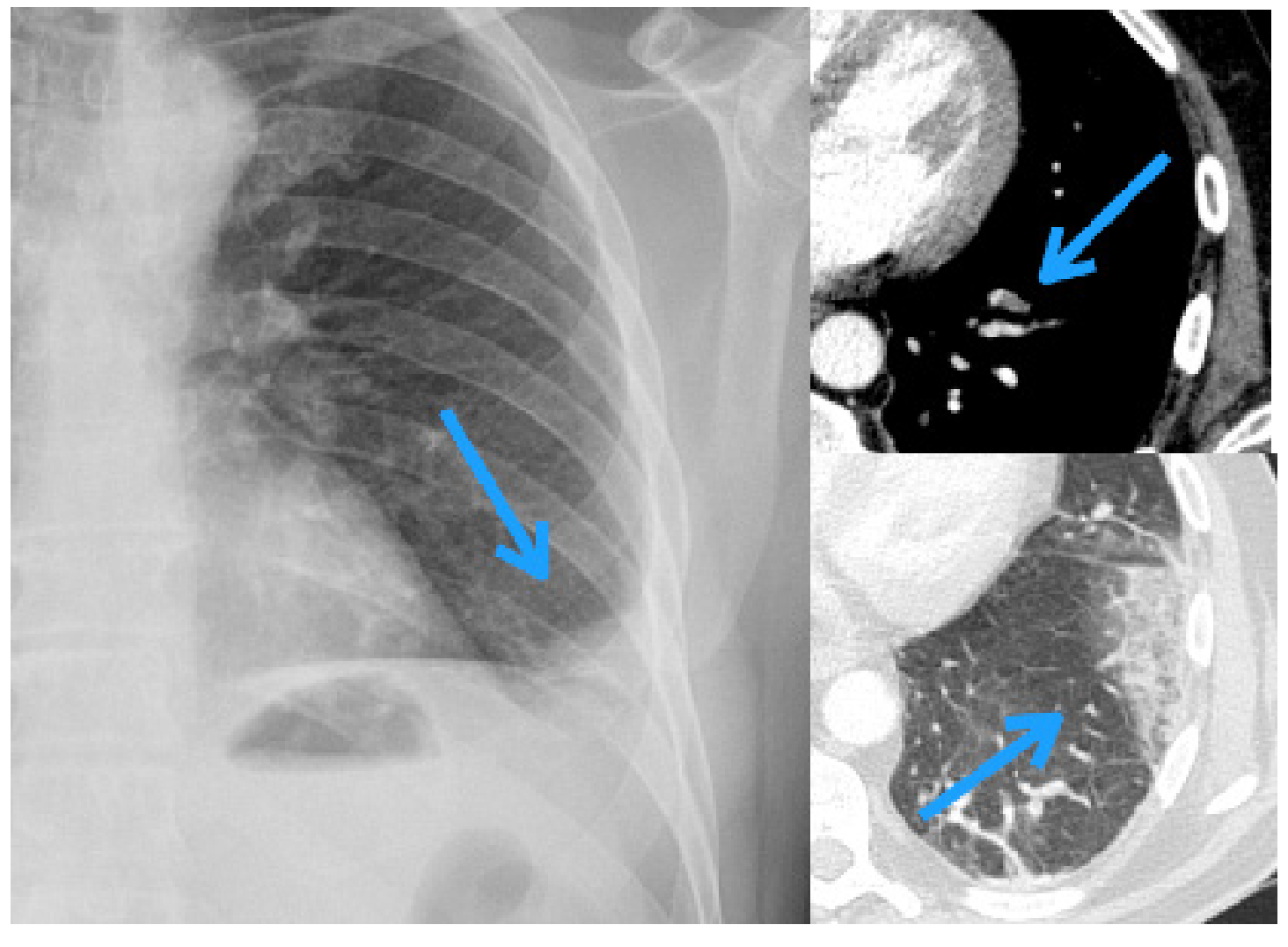

Figura 8: Paciente com TEP agudo em base pulmonar esquerda e imagem de infarto caracterizando o sinal da corcova de Hampton (seta). Na angioTC, identificamos o embolo (seta na imagem de janela mediastinal) e o infarto pulmonar correspondente (seta na imagem de janela pulmonar). 


\section{Dedo de luva}

Opacidade alongada e ramificada que representa uma bronquiectasia preenchida por conteúdo no interior, muitas vezes de impactação mucóide (retenção de secreção), em geral relacionado a uma obstrução proximal (por ex.: atresia brônquica, corpo estranho, tumor endoluminal) ou à aspergilose broncopulmonar alérgica no paciente asmático ${ }^{1,16}$ (figura 9).

\section{Diafragma contínuo}

Auxilia a identificação de pneumomediastino na radiografia em AP (anteroposterior) com o paciente deitado ou sentado, sendo sinal caracterizado por uma linha horizontal radiotransparente que atravessa a linha média superiormente ao diafragma, abaixo do coração ${ }^{19,20}$ (figura 10).

\section{Duplo contorno}

Indica aumento do átrio esquerdo na radiografia em PA, aparecendo como opacidade curvilínea na silhueta cardíaca direita medialmente ao contorno normal do átrio direito ${ }^{4,11}$ (figura 11). Também são sinais de aumento desta câmara cardíaca o deslocamento superior do brônquio fonte esquerdo e o surgimento do quarto arco cardíaco a esquerda.

\section{"Luftsichel"}

Sinal com nome na língua alemã que representa imagem de crescente aéreo adjacente ao arco aórtico na radiografia em PA, indicando colapso acentuado do lobo superior do pulmão esquerdo por diferentes patologias, inclusive por neoplasia obstrutiva central. A imagem radiotransparente se deve ao segmento superior do lobo inferior esquerdo hiperinsuflado e deslocado superiormente (hiperinsuflação compensatória) $)^{1,21-24}$ (figura 12).

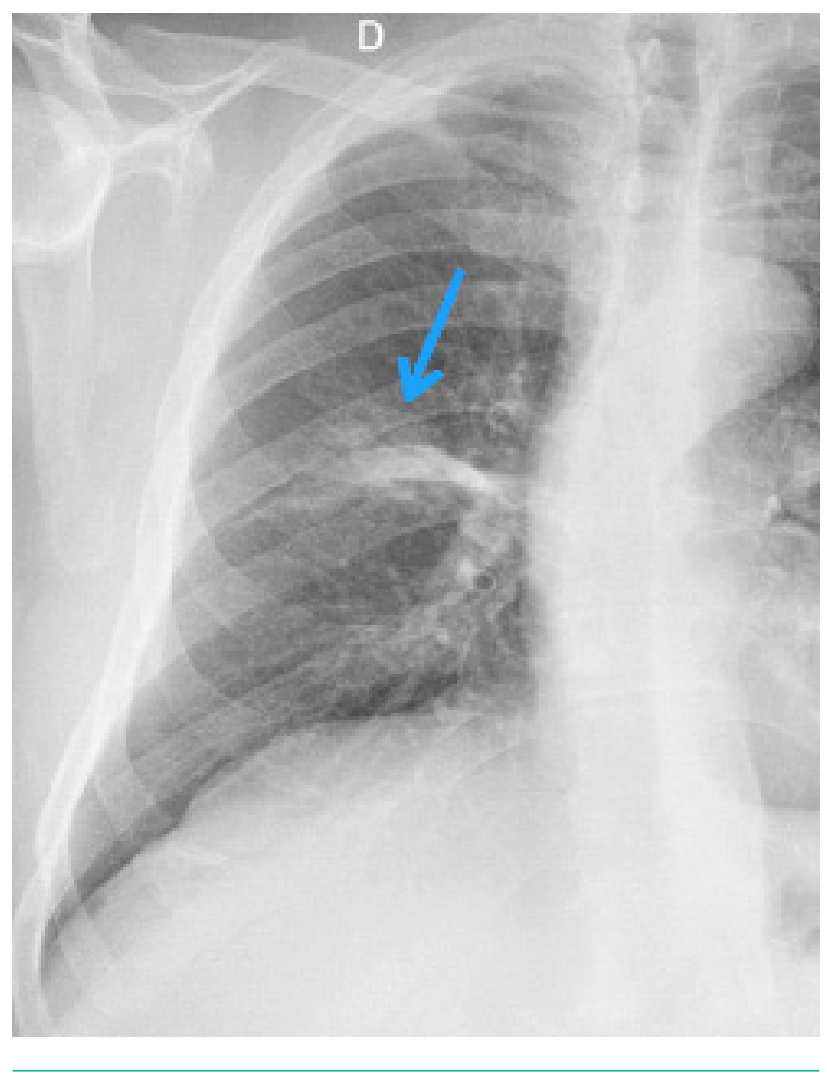

Figura 9: Sinal do dedo de luva em paciente asmático com aspergilose broncopulmonar alérgica.

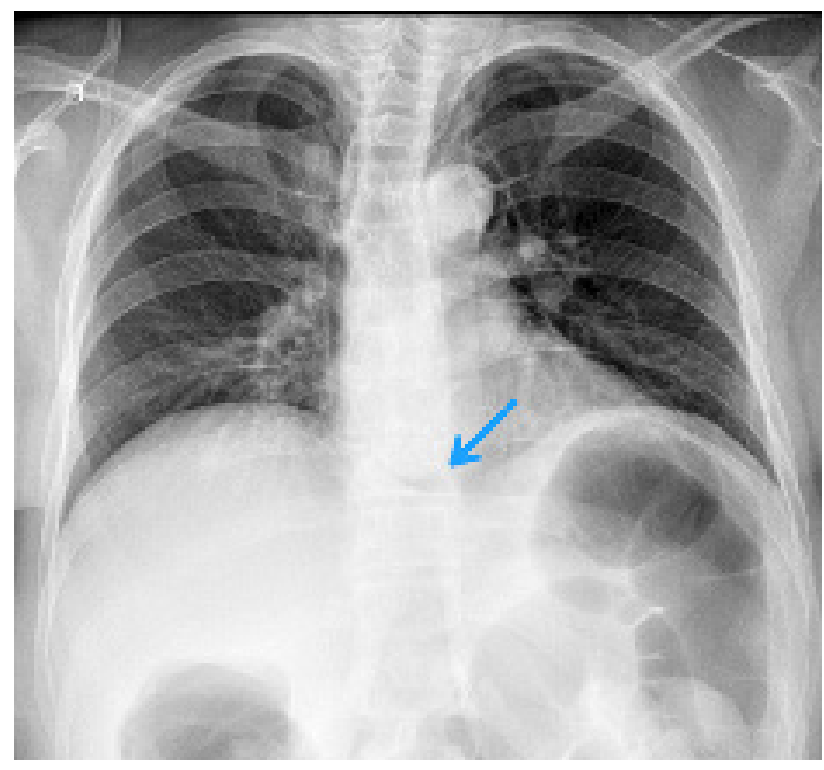

Figura 10: Radiografia AP de paciente internado com pneumomediastino e sinal do diafragma contínuo. 


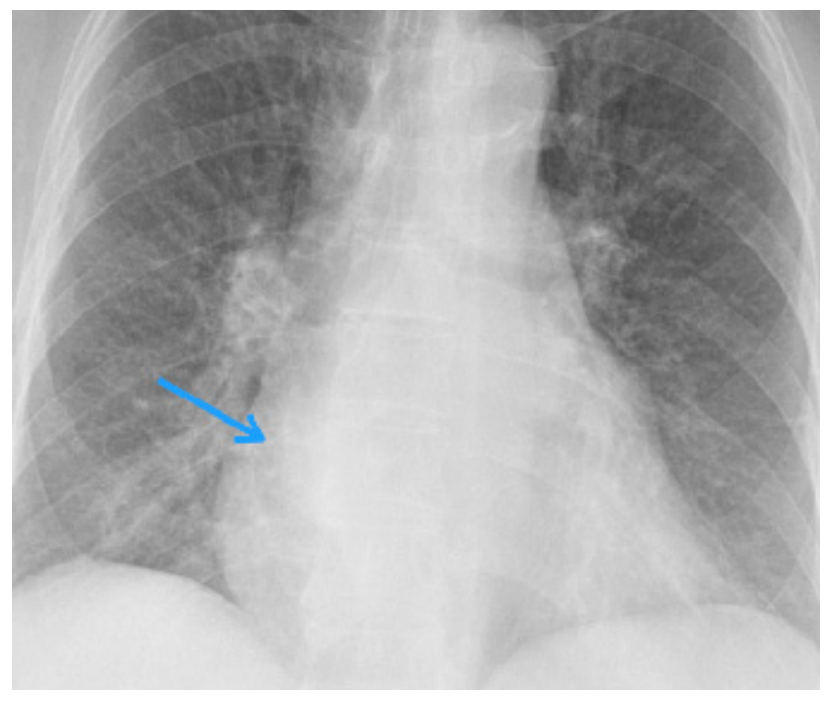

Figura 11: Paciente com doença mitral e sinais de dilatação do átrio esquerdo, incluindo o sinal do duplo contorno (seta), quarto arco esquerdo e luxação do brônquio fonte esquerdo.

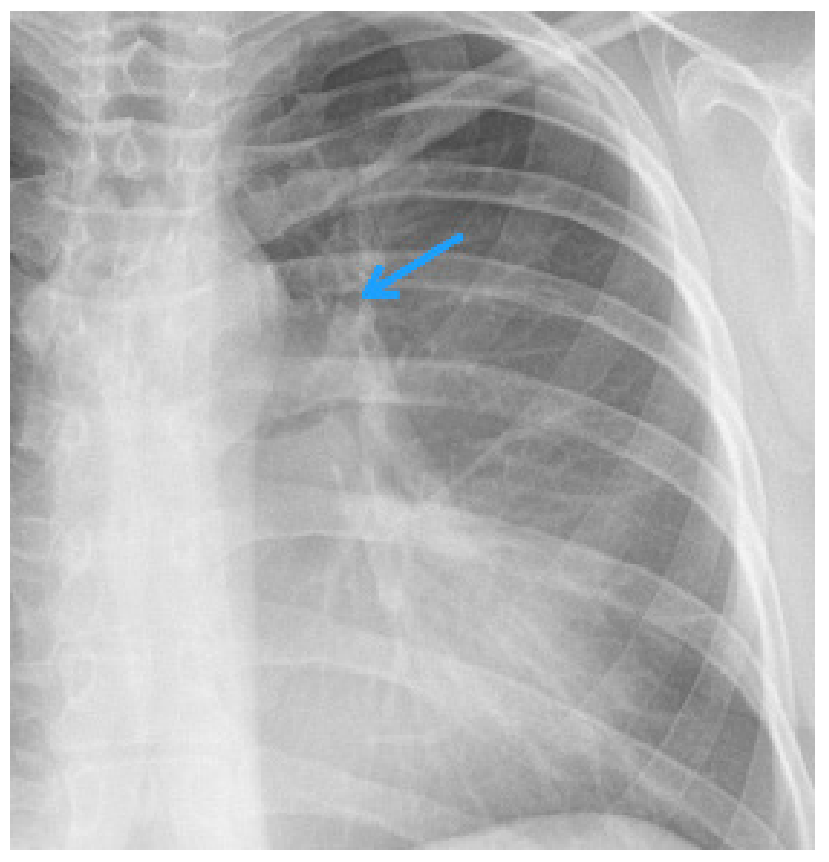

Figura 12: Sinal do "Luftsichel" em paciente com estenose brônquica e atelectasia do lobo superior do pulmão esquerdo.

\section{Menisco}

Representa a obliteração do seio costofrênico lateral ou posterior no RX, formando imagem de aspecto côncavo liso que, quando identificado, indica a presença de derrame pleural livre ${ }^{4,11}$ (figura 13).

\section{Palla}

Este sinal é representado pela dilatação de ramo da artéria pulmonar proximal a uma região de amputação vascular pulmonar (oligoemia), visto principalmente na artéria pulmonar descendente direita, podendo auxiliar na suspeição radiográfica de tromboembolismo pulmonar agudo $18,25,26$ (figura 14). O nome é homenagem a Antonio Palla (1950-), radiologista italiano.

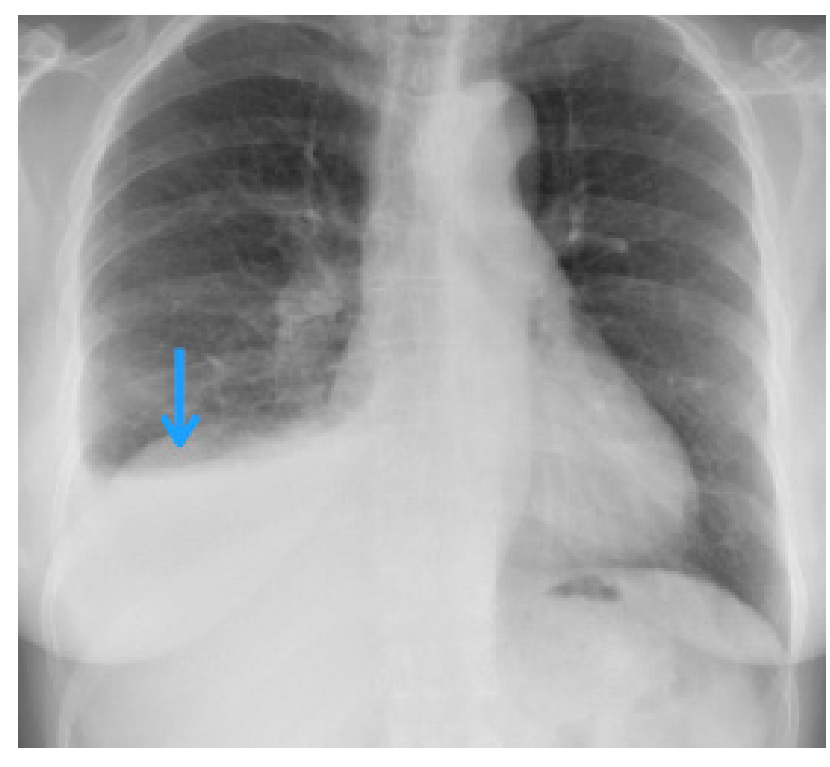

Figura 13: Derrame pleural livre a direita, com sinal do menisco.

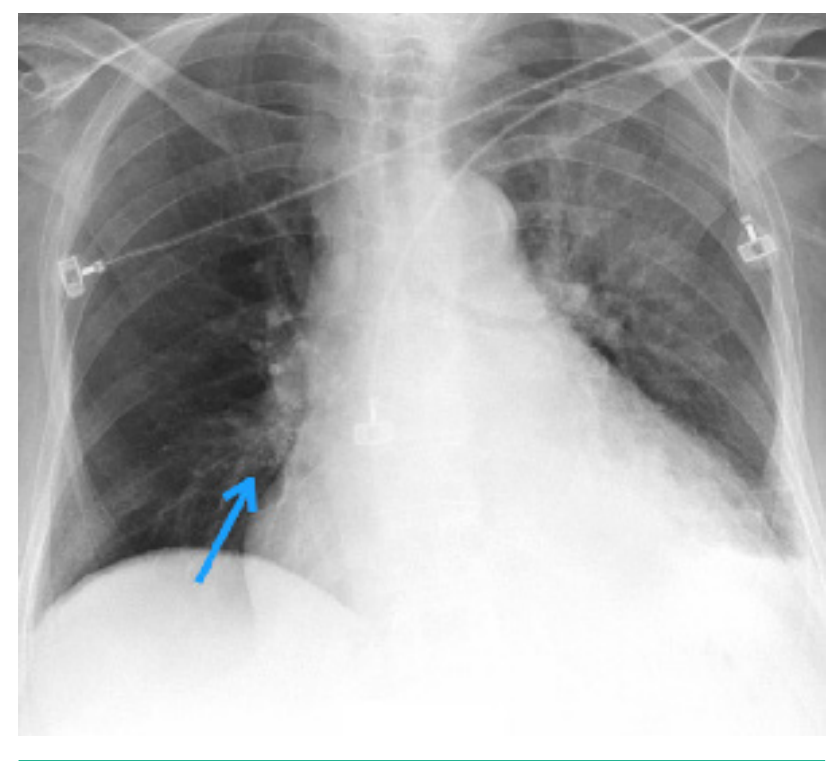

Figura 14: Paciente com extenso tromboembolismo pulmonar agudo a direita, notando-se hipertransparência do pulmão direito (sinal de Westermark) e dilatação com amputação da artéria descendente deste lado (sinal de Palla). 


\section{Pico justafrênico}

Imagem triangular que surge no aspecto mediano da cúpula diafragmática associada a atelectasia de lobo pulmonar superior, visto na radiografia em $\mathrm{PA}$. Este sinal é pouco específico e está relacionado a diferentes causas de atelectasia superior ${ }^{1,27}$ (figura 15).

\section{S de Golden}

O sinal do S de Golden, descrito por R. Golden em 1925, está relacionado a uma neoplasia central obstrutiva levando a atelectasia distal, mais comumente representando um carcinoma broncogênico. A porção côncava da opacidade (aspecto de $\mathrm{S}$ invertido) representa o contorno da massa e a porção convexa representa a atelectasia pós obstrutiva. Mais tipicamente é visto nos tumores do lobo superior do pulmão direito ${ }^{1,2}$ (figura 16).

\section{Silhueta}

Um dos primeiros sinais descritos em radiologia, indica a perda da definição da borda ou margem de uma estrutura ("perda da silhueta") que está em contato com opacidade de atenuação semelhante, em geral uma consolidação ou massa. O sinal da silhueta é importante pois nos ajuda a localizar a opacidade nas radiografias. Por exemplo, enquanto as opacidades do lobo médio e língula fazem sinal da silhueta com o coração, as opacidades dos lobos inferiores apagam as silhuetas diafragmáticas ${ }^{4,11}$ (figura 4).

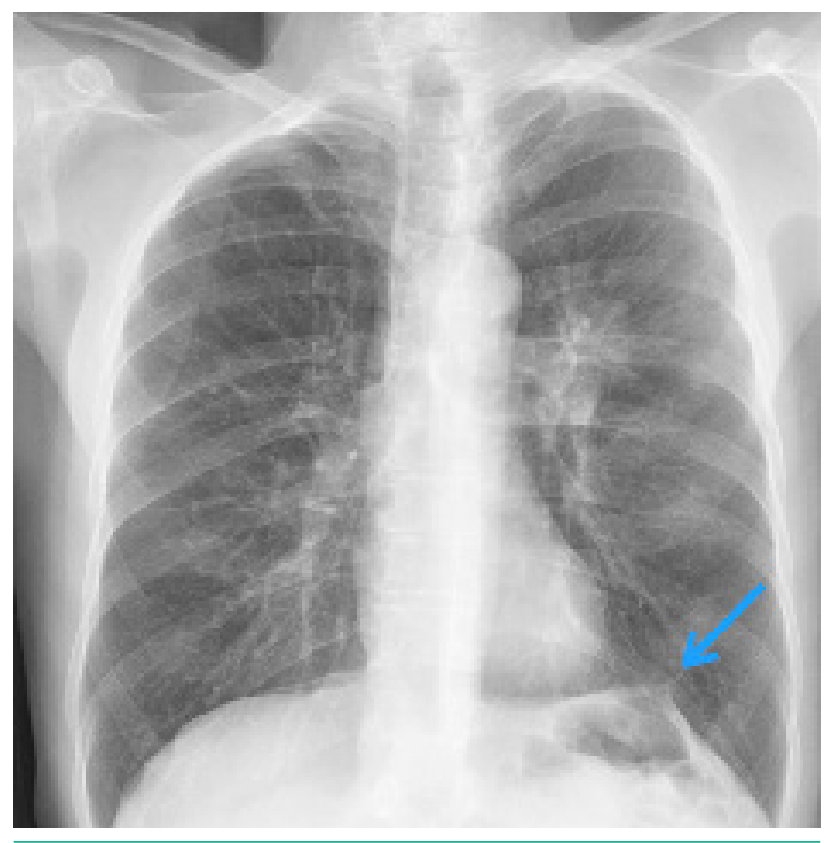

Figura 15: Sinal do pico epifrênico em paciente com câncer de pulmão central (células escamosas) envolvendo o brônquio lobar superior esquerdo, que perdeu volume.

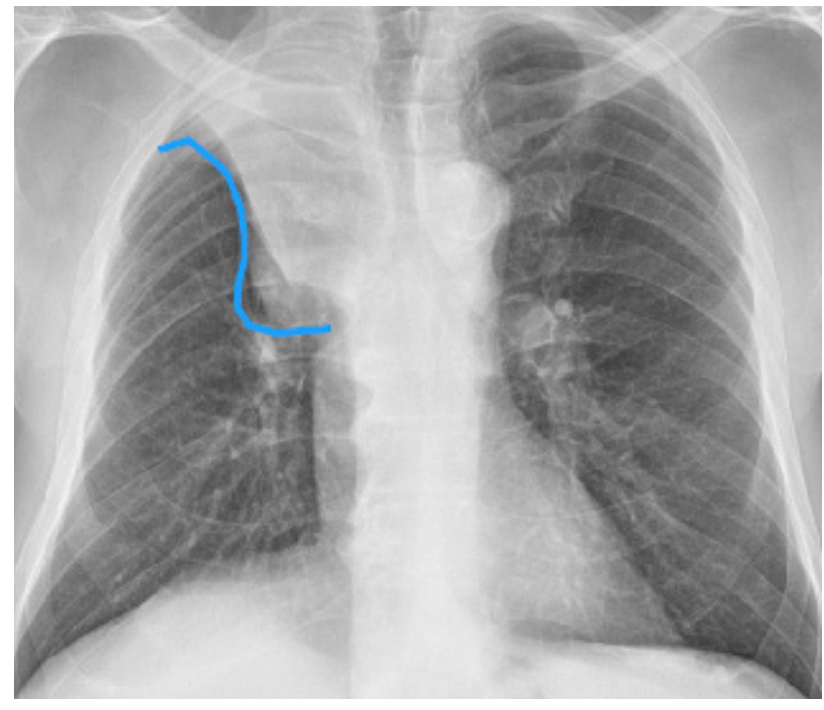

Figura 16: Paciente com neoplasia pulmonar central obstrutiva a direita e o sinal do $S$ de Golden na radiografia em PA. 


\section{Sobreposição hilar}

Sinal que indica, na radiografia em PA, que a opacidade projetada nesta região não tem origem nas estruturas do hilo pulmonar. Quando a lesão é mediastinal, pleural ou pulmonar, é possível observar os vasos normais do hilo pulmonar através da opacidade ${ }^{11,28,29}$ (figura 17).

\section{Sulco profundo}

Juntamente com o sinal do diafragma contínuo, auxilia na identificação do pneumotórax na radiografia em AP. O seio costofrênico lateral fica hipertransparente e tem aspecto mais profundo que o habitual ${ }^{30}$ (figura 10). É importante lembrar que para deteç̧ão do pneumotórax, o ideal é realizar o exame em PA com o paciente em ortostase, podendo também a grafia em expiração ajudar.

\section{Trilho de trem}

No RX de tórax, a imagem de bronquiectasia pode não ser característica, muitas vezes confundindo com opacidades intersticiais. O sinal do trilho de trem (linhas paralelas) representa a bronquiectasia vista no seu plano longitudinal e é a imagem radiográfica mais fidedigna para a presença de dilatação irreversível do brônquio ${ }^{31}$ (figura 18).

\section{"Westermark"}

Assim como o sinal de Palla, este sinal pode auxiliar na suspeição de tromboembolismo pulmonar agudo no RX. Aparece como uma região de hipertransparência focal na radiografia em PA, indicando área focal de oligoemia (figura 14). Homenagem ao radiologista sueco Nils Johan Hugo Westermark $^{1,26}$ (1892-1980).

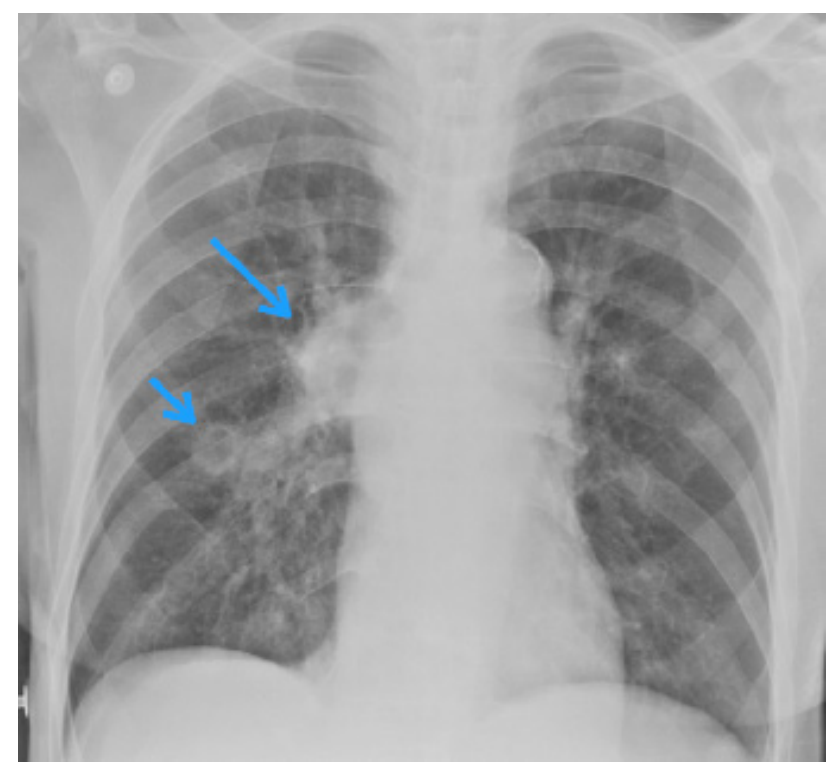

Figura 17: Sinal da sobreposição hilar, em um paciente com quadro infeccioso e imagem de abcesso pulmonar (segmento superior do lobo inferior) sobreposta ao hilo pulmonar direito na grafia em AP (seta). Há outro abcesso menor no mesmo pulmão (cavidade - seta curta).

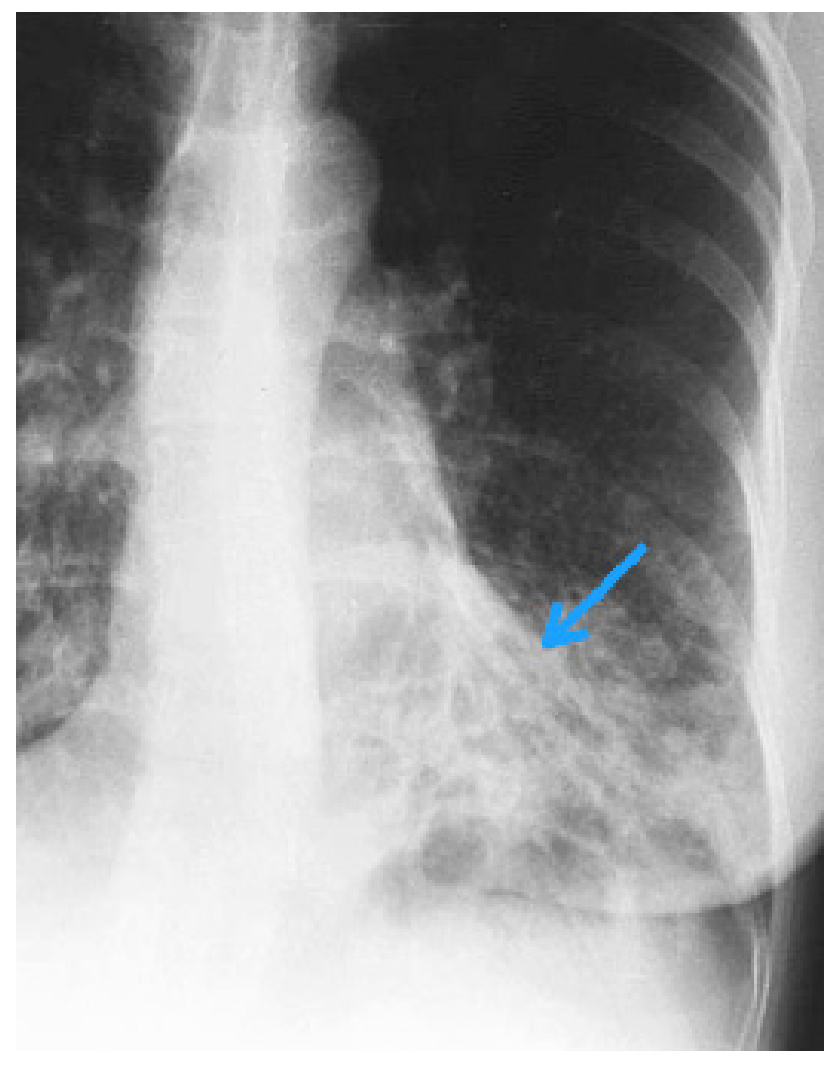

Figura 18: Bronquiectasias em lobo inferior do pulmão esquerdo, com sinal do trilho de trem. 


\section{CONCLUSÃO}

Neste texto foram apresentados e discutidos os seguintes temas:

- os principais sinais radiológicos na imagem torácica, sendo que alguns sinais são vistos no RX e na TC, enquanto outros em apenas um dos métodos

- a correlação dos sinais com algumas condições e doenças específicas, fortalecendo o conceito de que os sinais acrescentam especificidade à interpretação

\section{REFERÊNCIAS}

1. Marshall GB, Farnquist BA, MacGregor JH, Burrowes PW. Signs in Thoracic Imaging. J Thorac Imaging [Internet]. 2006;21(1):76-90. Disponível em: https://insights. ovid.com/crossref?an $=00005382-200603000-00020$

2. Algin O, Gokalp G, Topal U. Signs in chest imaging. Diagnostic Interv Radiol [Internet]. 2011;17(1):18-29. Disponível em: http://www.dirjournal.org/sayilar/34/ buyuk/pdf_DIR_324.pdf

3. Hansell DM, Bankier AA, MacMahon H, McLoud TC, Müller NL, Remy J. Fleischner Society: Glossary of Terms for Thoracic Imaging. Radiology [Internet]. 2008;246(3):697-722. Disponível em: http://radiology. rsna.org/content/246/3/697

4. Lacey G, Morley S, Berman L. Radiografia do Tórax - Um guia prático. Elsevier Editora Ltda; 2010.

5. Theros EG. RPC of the Month from the AFIP. Radiology [Internet]. 1969;92(7):1557-61. Disponível em: https://doi.org/10.1148/92.7.1557

6. Nunes H, Brillet P-Y, Valeyre D, Brauner MW, Wells AU. Imaging in Sarcoidosis. Semin Respir Crit Care Med [Internet]. 2007;28(1):102-20. Disponível em: http://www. thieme-connect.de/DOI/DOI?10.1055/s-2007-970336

7. Ouellette $\mathrm{H}$. The Signet Ring Sign. Radiology [Internet]. 1999;212(1):67-8. Disponível em: http://pubs.rsna.org/ doi/10.1148/radiology.212.1.r99j|2067

8. Gluecker T, Capasso P, Schnyder P, Gudinchet $F$, Schaller M-D, Revelly J-P, et al. Clinical and Radiologic Features of Pulmonary Edema. RadioGraphics [Internet]. 1999;19(6):1507-31. Disponível em: http://pubs.rsna.org/ doi/10.1148/radiographics.19.6.g99no211507

9. Hsu CC-T, Henry TS, Chung JH, Little BP. The Incomplete Border Sign. J Thorac Imaging [Internet]. 2014;29(4):W48. Disponível em: http://content.wkhealth.com/linkback/openurl?sid=WKPTLP:landingpage\&an=00005382-201407000-00011
10. Völk M, Strotzer M, Feuerbach S. Case of the month. Intrapulmonary or extrapulmonary? Br J Radiol [Internet]. 2000;73(868):451-2. Disponível em: http://www.birpublications.org/doi/10.1259/bjr.73.868.10844874

11. Goodman LR. Felson: princípios de radiologia do tórax: estudo dirigido. 2. ed. Atheneu; 2001.

12. Schneider HJ, Felson B, Gonzalez LL. Rounded atelectasis. Am J Roentgenol [Internet]. 1980;134(2):225-32. Disponível em: http://www.ajronline.org/doi/10.2214/ ajr.134.2.225

13. Felson B. More Chest Roentgen Signs and How to Teach Them. Radiology [Internet]. 1968;90(3):429-41. Disponível em: http://pubs.rsna.org/doi/10.1148/90.3.429

14. HALASZ NA, HALLORAN KH, LIEBOW AA. Bronchial and arterial anomalies with drainage of the right lung into the inferior vena cava. Circulation [Internet]. 1956;14(5):826-46. Disponível em: http://www.ncbi.nlm.nih.gov/pubmed/ 13374858

15. Walker CM, Abbott GF, Greene RE, Shepard J-AO, Vummidi D, Digumarthy SR. Imaging Pulmonary Infection: Classic Signs and Patterns. Am J Roentgenol [Internet]. 2014;202(3):479-92. Disponível em: http://www. ajronline.org/doi/10.2214/AJR.13.11463

16. Franquet $T$, Müller NL, Giménez $A$, Guembe $P$, Torre J de La, Bagué S. Spectrum of Pulmonary Aspergillosis: Histologic, Clinical, and Radiologic Findings. Radiographics [Internet]. 2001;21(4):825-37. Disponível em: http://pubs.rsna.org/doi/10.1148/radiographics.21.4. g01j103825

17. Hampton A, Castleman B. Correlation of postmortem chest teleroentgenograms with autopsy findings with special reference to pulmonary embolism and infarction. AJR Am J Roentgenol. 1940;43:305-26.

18. Dalen JE. Pulmonary embolism: what have we learned since Virchow? Natural history, pathophysiology, and diagnosis. Chest [Internet]. 2002;122(4):1440-56. Disponível em: http://www.ncbi.nlm.nih.gov/pubmed/ 12377877

19. Levin B. The continuous diaphragm sign. A newly-recognized sign of pneumomediastinum. Clin Radiol [Internet]. 1973;24(3):337-8. Disponível em: http://www. ncbi.nlm.nih.gov/pubmed/4730225

20. Zylak CM, Standen JR, Barnes GR, Zylak CJ. Pneumomediastinum Revisited. Radiographics [Internet]. 2000;20(4):1043-57. Disponível em: http://pubs.rsna. org/doi/10.1148/radiographics.20.4.g00jl131043

21. Godoy MCB, Viswanathan C, Marchiori E, Truong MT, Benveniste MF, Rossi $S$, et al. The reversed halo sign: update and differential diagnosis. $\mathrm{Br}$ J Radiol [Internet]. 2012;85(1017):1226-35. Disponível em: http://www. birpublications.org/doi/10.1259/bjr/54532316

22. Chong BJ, Kanne JP, Chung JH. Headcheese Sign. J Thorac Imaging [Internet]. 2014;29(1):W13. Disponível em: http://content.wkhealth.com/linkback/ openurl ?sid =WKPTLP: landing page $\& a n=00005382$ 201401000-00012 
23. Webb WR. Thin-Section CT of the Secondary Pulmonary Lobule: Anatomy and the Image-The 2004 Fleischner Lecture. Radiology [Internet]. 2006;239(2):322-38. Disponível em: http://pubs.rsna.org/doi/10.1148/ radiol.2392041968

24. Webber M, Davies P. The Luftsichel: an old sign in upper lobe collapse. Clin Radiol [Internet]. 1981;32(3):271-5. Disponível em: http://www.ncbi.nlm.nih.gov/pubmed/7237906

25. Palla A, Donnamaria V, Petruzzelli S, Rossi G, Riccetti G, Giuntini C. Enlargement of the right descending pulmonary artery in pulmonary embolism. Am J Roentgenol [Internet]. 1983;141(3):513-7. Disponível em: http:// www.ajronline.org/doi/10.2214/ajr.141.3.513

26. Sreenivasan S, Bennett S, Parfitt VJ. Westermark's and Palla's Signs in Acute Pulmonary Embolism. Circulation [Internet]. 2007;115(8). Disponível em: https://www.ahajournals.org/doi/10.1161/CIRCULATIONAHA.106.665422
27. Kattan KR, Eyler WR, Felson B. The juxtaphrenic peak in upper lobe collapse. Radiology [Internet]. 1980;134(3):763-5. Disponível em: http://pubs.rsna.org/doi/10.1148/radiology.134.3.7355230

28. Felson $\mathrm{B}$, Felson $\mathrm{H}$. Localization of Intrathoracic Lesions by Means of the Postero-Anterior Roentgenogram. Radiology [Internet]. 1950;55(3):363-74. Disponível em: http://pubs.rsna.org/doi/10.1148/55.3.363

29. Whitten CR, Khan S, Munneke GJ, Grubnic S. A Diagnostic Approach to Mediastinal Abnormalities. Radiographics. 2007;27(3):657-71.

30. Gordon R. The deep sulcus sign. Radiology [Internet]. 1980 Jul;136(1):25-7. Disponível em: http://pubs.rsna.org/ doi/10.1148/radiology.136.1.7384513

31. Cantin L, Bankier AA, Eisenberg RL. Bronchiectasis. Am J Roentgenol [Internet]. 2009;193(3):W158-71. Disponível em: http://www.ajronline.org/doi/10.2214/AJR.09.3053 


\section{MATERIAL SUPLEMENTAR}

\section{EXERCÍCIOS}

1. São sinais relacionados ao tromboembolismo pulmonar agudo, exceto:

a) Corcova de Hampton

b) Westermark

c) Palla

d) Luftsichel

e) Todos são sinais relacionados ao TEP agudo

2. Qual o sinal que pode auxiliar na identificação do pneumotórax em uma radiografia em AP?

3. Qual o sinal identificado na imagem em PA deste exame de RX de tórax? Qual a provável alteração?

4. Qual a diferença de significado entre os sinais da convergência e da sobreposição hilar?

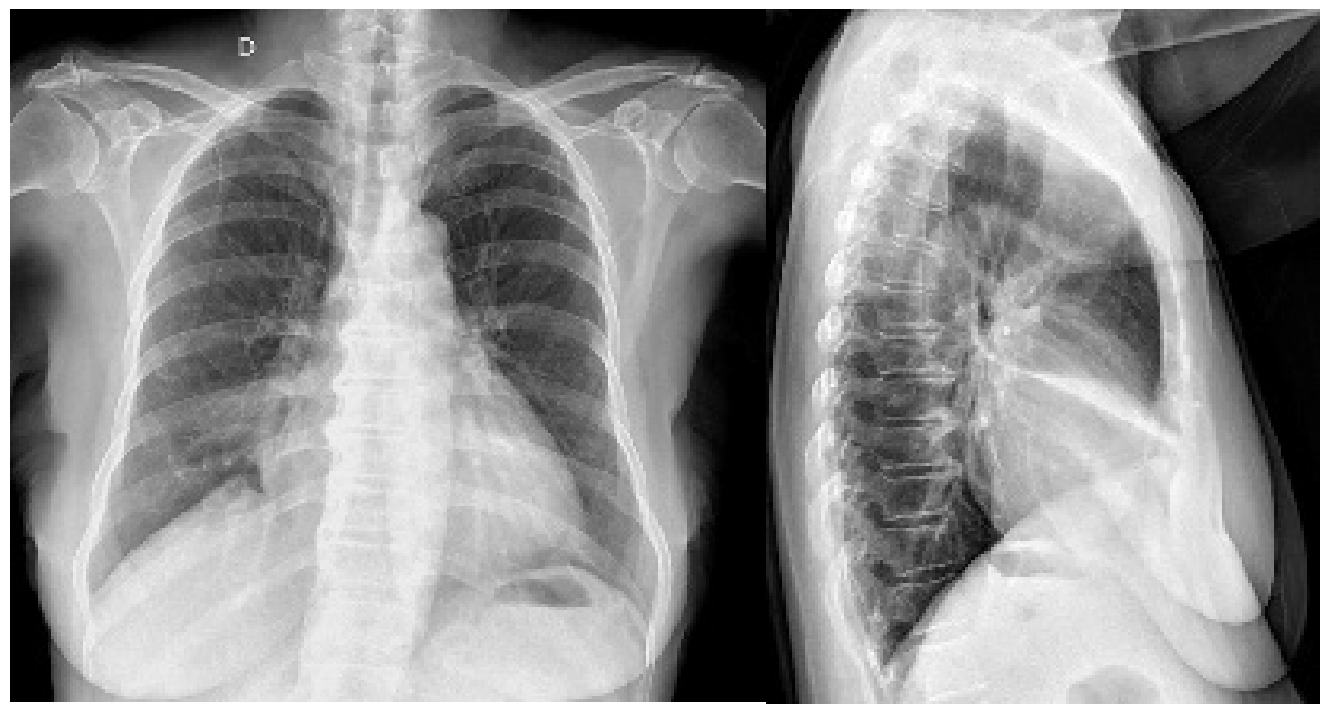

5. Correlacione cada sinal com sua descrição ou significado.

A. Sinal do 1-2-3

( ) Atelectasia redonda

B. Sinal da cauda de cometa

( ) Neoplasia central obstrutiva

C. S de Golden

( ) Bola fúngica

D. Split pleura

E. Sinal do crescente aéreo

( ) Linfonodopatia paratraqueal e hilar bilateral

( ) Empiema 